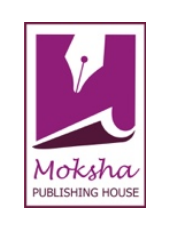

\author{
Review Article \\ www.ijrap.net (ISSN:2229-3566)
}

\title{
FORMULATIONS OF GHRITA IN CHARAKA SAMHITA: A SYNOPTIC VIEW
}

Anubha Yadav *

PG Scholar, Department of Rasashastra and Bhaishajya Kalpana, All India Institute of Ayurveda, New Delhi, India

Received on: 28/12/19 Accepted on: 15/02/20

\author{
*Corresponding author \\ E-mail: dr.anubhayadav@gmail.com
}

DOI: $10.7897 / 2277-4343.110244$

\begin{abstract}
Ayurveda can be defined as a system, which uses principles of nature to promote, preserve health and eradicate diseases. This system focuses on keeping body, mind and spirit of an individual in equilibrium with nature. The Materia Medica of Ayurveda comprises of resources of plant, animal, metal and mineral origin, which have been advocated for use in different pathologies. In Ayurveda, drug therapy has great importance. Besides the five basic formulations viz. 'Swarasa', 'Kalka', 'Kwatha', 'Hima' and 'Phanta', there are many secondary preparations, descriptions of which is comprehensively explained in the classics. Medicated oils and Ghee are one such important category; where ghee is boiled with prescribed decoctions and paste of drugs. This process possibly ensures absorption of the active therapeutic properties of the ingredients into the oleaginous base. In such oleaginous preparations, three ingredients are essential - (1) ghee, (2) liquid, (3) fine paste of the ingredients. Description of standard methods of preparation, different stages of cooking, ideal characters of good preparations, utilization, limitations, dose, mode of utilization etc. have been explained systematically in the literature. Considering the importance of Ghrita Kalpana; a large number of formulations have been mentioned in Charaka Samhita. As no information is available on the numbers of Ghrita formulations in the classical literature; an attempt has been made to review Charaka Samhita and place information at one place.
\end{abstract}

Keywords: Ayurveda, Charaka Samhita, Ghrita

\section{INTRODUCTION}

Ayurveda can be defined as a system, which uses principles of nature to promote, preserve health and eradicate diseases. This system focuses on keeping body, mind and spirit of an individual in equilibrium with nature. It is systematically documented oldest medical system of the world is existing in Indian scenario since time immemorial. This science influenced many other medical systems of the world, hence, is referred as Mother of all sciences. Rigveda and Atharvaveda, the most ancient compendia of Indian culture and wisdom replete with references of health, diseases and treatment with natural resources, which subsequently developed into an organized knowledge called Samhitas. The Charaka Samhita is the first recorded treatise fully devoted to the concepts and practice of Ayurveda; its primary focus was therapeutics. It is an important compendium of Ayurveda containing systematic description of fundamental principles, basic concepts, causation and genesis of diseases, diagnosis, prognosis, modes and modalities of treatment etc. This system is attracting attention of developing countries as an alternative or adjuvant to synthetic drugs. The science is not merely a compendium of drugs but, is actually a way of life, perhaps is partly medical and partly social, cultural and laid down definite laws of healthy-living, personal cleanliness, good dietetic habits etc., is something which India has to be proud of and make every effort to retain and popularize it.

The Materia Medica of Ayurveda comprises of resources of plant, animal, metal and mineral origin, which have been advocated for use in different pathologies. Often, these resources have been used as ingredients of poly-herbal, herbo-mineral and metallic compound formulations by the seers, who have documented their clinical experiences and passed on the knowledge to further generations.

In Ayurveda, drug therapy has great importance. The formulations are developed based on classical fundamental principles and five basic formulations have been explained viz. 'Swarasa', 'Kalka', 'Kwatha', 'Hima' and 'Phanta'. Besides these, there are many secondary preparations, descriptions of which are comprehensively described in the classics. Medicated oils and Ghee are one such important category, where oil or ghee is boiled with prescribed decoctions and paste of drugs. This process possibly ensures absorption of the active therapeutic properties of the ingredients used, into the oleaginous base. In such oleaginous preparations, three ingredients are essential - (1) ghee or oil, (2) liquid, (3) fine paste of theingredients ${ }^{2}$. In general, oil will be used in four parts, one part of paste and sixteen parts of liquid ${ }^{3}$ (with several exceptions). Description of standard methods of preparation, different stages of cooking, ideal characters of good preparations, utilization limitations, dose, mode of utilization etc. have been explained systematically in the literature.

Considering the importance of Ghrita Kalpana; a number of formulations have been mentioned in Charaka Samhita. As no information is available on the numbers of Ghrita formulations in the classical literature; an attempt has been made to review Charaka Samhita ${ }^{4}$ and gather and place information at one place.

The Ghrita Kalpanas found in the management of different diseases is placed at Table 1 and Graph 1 . 
Anubha Yadav / Int. J. Res. Ayurveda Pharm. 11 (2), 2020

Table 1: Disease-wise list of Ghrita Kalpanas

\begin{tabular}{|c|c|c|}
\hline & Ghrita Kalpanas & References \\
\hline \multicolumn{3}{|c|}{ Rasayana Chikitsa } \\
\hline 1. & Amalaka Ghrita & Ch. Chi. 1-2/4 \\
\hline \multicolumn{3}{|c|}{ Vajikaran Chikitsa } \\
\hline 1. & Vajikaran Ghrita & Ch. Chi. 2-1/35 \\
\hline 2. & Vrishya Ghrita & Ch. Chi. 2-2/21-22 \\
\hline 3. & Vrishya Shatavari Ghrita & Ch. Chi. 2-3/18 \\
\hline 4. & Apatyakara Ghrita & Ch. Chi. 2-4/25-26 \\
\hline 5. & Apatyakara Ghrita II & Ch. Chi. 2-4/28-29 \\
\hline \multicolumn{3}{|c|}{ Jwara Chikitsa } \\
\hline 1. & Pippalyadi Ghrita & Ch. Chi. 3/219-220 \\
\hline 2. & Vasadi Ghrita & Ch. Chi. 3/222-223 \\
\hline 3. & Baladi Ghrita & Ch. Chi. $3 / 226$ \\
\hline 4. & Sahasradhouta Ghrita & Ch. Chi. 3/257 \\
\hline 5. & Shatpala Ghrita & Ch. Chi. 3/298 \\
\hline \multicolumn{3}{|c|}{ Raktapitta Chikitsa } \\
\hline 1. & Vasa Ghrita & Ch. Chi. 4/88 \\
\hline 2. & Shatarvaryadi Ghrita & Ch. Chi. 4/95 \\
\hline 3. & Panchapanchamoola Ghrita & Ch. Chi. 4/96 \\
\hline \multicolumn{3}{|c|}{ Gulma Chikitsa } \\
\hline 1. & Tilavaka Ghrita & Ch. Chi. 5/35 \\
\hline 2. & Trayushanadi Ghrita I \& II & Ch. Chi. 5/65-66 \\
\hline 3. & Hingusovarchaladya Ghrita & Ch. Chi. 5/69-70 \\
\hline 4. & Hapushadya Ghrita & Ch. Chi. 5/71-72 \\
\hline 5. & Pippalayadi Ghrita & Ch. Chi. 5/74 \\
\hline 6. & Nilini Ghrita & Ch. Chi. 5/105 \\
\hline 7. & Nilinadya Ghrita & Ch. Chi. 5/106-107 \\
\hline 8. & Rohinayadya Ghrita & Ch. Chi. 5/115-116 \\
\hline 9. & Trayamanadya Ghrita & Ch. Chi. 5/118-120 \\
\hline 10. & Amalakadya Ghrita & Ch. Chi. 5/122 \\
\hline 11. & Drakshadya Ghrita & Ch. Chi. 5/123-124 \\
\hline 12. & Vasa Ghrita & Ch. Chi. 5/126-127 \\
\hline 13. & Dashmooli Ghrita & Ch. Chi. 5/142 \\
\hline 14. & Bhallatakadya Ghrita & Ch. Chi. 5/143-145 \\
\hline 15. & Ksheera Shatpala Ghrita & Ch. Chi. 5/147-148 \\
\hline \multicolumn{3}{|c|}{ Prameha Chikitsa } \\
\hline 1. & Trikantakadya Sneha Ghrita & Ch. Chi. 6/38-39 \\
\hline \multicolumn{3}{|c|}{ Kushtha Chikitsa } \\
\hline 1. & Vipadikahar Ghrita-Taila & Ch. Chi. $7 / 120$ \\
\hline 2. & Tikta Ghrita & Ch. Chi. $7 / 134$ \\
\hline 3. & Khadir Ghrita & Ch. Chi. 7/135 \\
\hline 4. & Nimba Ghrita & Ch. Chi. $7 / 135$ \\
\hline 5. & Darvi Ghrita & Ch. Chi. 7/135 \\
\hline 6. & Patola Ghrita & Ch. Chi. $7 / 135$ \\
\hline 7. & Tiktashatpalaka Ghrita & Ch. Chi. 7/140-142 \\
\hline 8. & Mahatiktaka Ghrita & Ch. Chi. 7/144-150 \\
\hline 9. & Mahakhadir Ghrita & Ch. Chi. 7/152-156 \\
\hline \multicolumn{3}{|c|}{ Rajayakshama Chikitsa } \\
\hline 1. & Dashmoolayadya Ghrita & Ch. Chi. 8/93 \\
\hline 2. & Rasna Ghrita & Ch. Chi. 8/94 \\
\hline 3. & Bala Ghrita & Ch. Chi. 8/94 \\
\hline 4. & Kharjuradi Ghrita & Ch. Chi. 8/96 \\
\hline 5. & Dashmoola Ghrita & Ch. Chi. 8/97 \\
\hline 6. & Pancha Panchamoola Ghrita & Ch. Chi. 8/99 \\
\hline 7. & Duralbhadya Ghrita & Ch. Chi. 8/106-110 \\
\hline 8. & Jivantyadi Ghrita & Ch. Chi. 8/111-113 \\
\hline 9. & Mansa-sadhit Ghrita & Ch. Chi. 8/167 \\
\hline 10. & Panchkoladi Ghrita & Ch. Chi. 8/169-170 \\
\hline 11. & Rasna Ghrita & Ch. Chi. 8/170-171 \\
\hline \multicolumn{3}{|c|}{ Unmada Chikitsa } \\
\hline 1. & Hingvadya Ghrita & Ch. Chi. 9/34 \\
\hline 2. & Kalyanaka Ghrita & Ch. Chi. 9/35-41 \\
\hline 3. & Mahakalyanaka Ghrita & Ch. Chi. 9/42-44 \\
\hline 4. & Mahapaishachika Ghrita & Ch. Chi. 9/45-48 \\
\hline 5. & Lashunadya Ghrita & Ch. Chi. 9/49-51 \\
\hline 6. & Lashunadya Ghrita II & Ch. Chi. 9/52-56 \\
\hline 7. & Purana Ghrita & Ch. Chi. 9/59 \\
\hline 8. & Siddharthaka Ghrita & Ch. Chi. 9/72 1/2 \\
\hline
\end{tabular}


Anubha Yadav / Int. J. Res. Ayurveda Pharm. 11 (2), 2020

\begin{tabular}{|c|c|c|}
\hline \multicolumn{3}{|c|}{ Apasmara Chikitsa } \\
\hline 1. & Panchagavya Ghrita & Ch. Chi. $10 / 17$ \\
\hline 2. & Mahapanchagavya Ghrita & Ch. Chi. 10/18-24 \\
\hline 3. & Brahmi Ghrita & Ch. Chi. $10 / 25$ \\
\hline 4. & Vachadi Ghrita & Ch. Chi. $10 / 27$ \\
\hline 5. & Yamaka & Ch. Chi. $10 / 28$ \\
\hline 6. & Amalakadi Ghrita & Ch. Chi. $10 / 31$ \\
\hline \multicolumn{3}{|c|}{ Kshataksheena Chikitsa } \\
\hline 1. & Amritprash Ghrita & Ch. Chi. 11/35-43 \\
\hline 2. & Shvadanshtradi Ghrita & Ch. Chi. 11/44-47 \\
\hline \multicolumn{3}{|c|}{ Shwayathu Chikitsa } \\
\hline 1. & Chitrakadi Ghrita & Ch. Chi. $12 / 55-56$ \\
\hline 2. & Dwitiya Chitrakadi Ghrita & Ch. Chi. $12 / 57$ \\
\hline 3. & Chitraka Ghrita & Ch. Chi. $12 / 58-59$ \\
\hline \multicolumn{3}{|c|}{ Udara Chikitsa } \\
\hline 1. & Rohitaka Ghrita & Ch. Chi. 13/83-85 \\
\hline 2. & Panchakola Ghrita & Ch. Chi. 13/112-113 \\
\hline 3. & Nagaradi Ghrita & Ch. Chi. 13/115 \\
\hline 4. & Chitraka Ghrita & Ch. Chi. 13/116-117 \\
\hline 5. & Yavadi Ghrita & Ch. Chi. 13/117-118 \\
\hline 6. & Snuhiksheera Ghrita & Ch. Chi. $13 / 138-140$ \\
\hline \multicolumn{3}{|c|}{ Arsha Chikitsa } \\
\hline 1. & Pippalyadi Ghrita & Ch. Chi. 14/104 \\
\hline 2. & Apar Pippalyadi Ghrita & Ch. Chi. 14/106 \\
\hline 3. & Chavyadi Ghrita & Ch. Chi. 14/107-109 \\
\hline 4. & Nagaradya Ghrita & Ch. Chi. $14 / 110-112$ \\
\hline 5. & Tritiya Pippalyadi Ghrita & Ch. Chi. 14/113-118 \\
\hline 6. & Kutajaphaladi Ghrita & Ch. Chi. 14/197 \\
\hline 7. & Hriberadi Ghrita & Ch. Chi. $14 / 230-233$ \\
\hline 8. & Sunnishannaka changeri Ghrita & Ch. Chi. 14/234-242 \\
\hline \multicolumn{3}{|c|}{ Grahani Chikitsa } \\
\hline 1. & Dashmooladya Ghrita & Ch. Chi. $15 / 82-86$ \\
\hline 2. & Tryushanadya Ghrita & Ch. Chi. 15/87 \\
\hline 3. & Panchamooladya Ghrita & Ch. Chi. 15/88-91 \\
\hline 4. & Chandanadya Ghrita & Ch. Chi. $15 / 125-128$ \\
\hline 5. & Kshara Ghrita & Ch. Chi. $15 / 171-172$ \\
\hline \multicolumn{3}{|c|}{ Panduroga Chikitsa } \\
\hline 1. & Dadima Ghrita & Ch. Chi. 16/ 44-46 \\
\hline 2. & Katukadya Ghrita & Ch. Chi. 16/47-49 \\
\hline 3. & Pathya Ghrita & Ch. Chi. $16 / 50$ \\
\hline 4. & Danti Ghrita & Ch. Chi. $16 / 51$ \\
\hline 5. & Draksha Ghrita & Ch. Chi. 16/52 \\
\hline 6. & Haridradi Ghrita & Ch. Chi. 16/53 \\
\hline 7. & Vyoshadya Ghrita & Ch. Chi. 16/119-120 \\
\hline \multicolumn{3}{|c|}{ Hikkashwas Chikitsa } \\
\hline 1. & Dashmooladi Ghrita & Ch. Chi. $17 / 140$ \\
\hline 2. & Tejovatyadi Ghrita & Ch. Chi. 17/141-144 \\
\hline 3. & Manahshiladi Ghrita & Ch. Chi. 17/145 \\
\hline \multicolumn{3}{|c|}{ Kasa Chikitsa } \\
\hline 1. & Kantakari Ghrita & Ch. Chi. $18 / 35$ \\
\hline 2. & Pippalyadi Ghrita & Ch. Chi. $18 / 36-38$ \\
\hline 3. & Triushnadi Ghrita & Ch. Chi. $18 / 39-42$ \\
\hline 4. & Rasna Ghrita & Ch. Chi. 18/43-46 \\
\hline 5. & Dashmooladi Ghrita & Ch. Chi. 18/123-124 \\
\hline 6. & Kantakari Ghrita & Ch. Chi. $18 / 125-128$ \\
\hline 7. & Kulatthadi Ghrita & Ch. Chi. $18 / 129$ \\
\hline 8. & Dwipanchamooladi Ghrita & Ch. Chi. $18 / 158-160$ \\
\hline 9. & Guduchyadi Ghrita & Ch. Chi. 18/161-162 \\
\hline 10. & Kasamardadi Ghrita & Ch. Chi. $18 / 163-164$ \\
\hline \multicolumn{3}{|c|}{ Atisara Chikitsa } \\
\hline 1. & Changeri Ghrita & Ch. Chi. 19/43 \\
\hline 2. & Chavyadi Ghrita & Ch. Chi. $19 / 44$ \\
\hline 3. & Darvyadi Ghrita & Ch. Chi. $19 / 80-81$ \\
\hline 4. & Shatavari Ghrita & Ch. Chi. 19/97 \\
\hline 5. & Nyogrodhadi Ghrita & Ch. Chi. 19/99-100 \\
\hline \multicolumn{3}{|c|}{ Chardi Chikitsa } \\
\hline 1. & Kalyanaka Ghrita & Ch. Chi. $20 / 47$ \\
\hline 2. & Triushnadi Ghrita & Ch. Chi. 20/47 \\
\hline 3. & Jeevaniya Ghrita & Ch. Chi. $20 / 47$ \\
\hline \multicolumn{3}{|c|}{ Visarpa Chikitsa } \\
\hline 1. & Tikta Ghrita & Ch. Chi. 21/47 \\
\hline
\end{tabular}


Anubha Yadav / Int. J. Res. Ayurveda Pharm. 11 (2), 2020

\begin{tabular}{|c|c|c|}
\hline \multicolumn{3}{|c|}{ Trishna Chikitsa } \\
\hline 1. & Jeevaniya Ghrita & Ch. Chi. 22/41 \\
\hline \multicolumn{3}{|c|}{ Visha Chikitsa } \\
\hline 1. & Nagdantyadi Ghrita & Ch. Chi. 23/241 \\
\hline 2. & Amrita Ghrita & Ch. Chi. 23/242-249 \\
\hline \multicolumn{3}{|c|}{ Vrana Chikitsa } \\
\hline 1. & Shatadhauta Ghrita & Ch. Chi. 25/64 \\
\hline \multicolumn{3}{|c|}{ Udavarta Chikitsa } \\
\hline 1. & Sthiradi Ghrita & Ch. Chi. 26/23 \\
\hline \multicolumn{3}{|c|}{ Ashmari Chikitsa } \\
\hline 1. & Swadanstradi Ghrita & Ch. Chi. 26/74 \\
\hline \multicolumn{3}{|c|}{ Hridroga Chikitsa } \\
\hline 1. & Haritakyadi Ghrita & Ch. Chi. 26/83 \\
\hline 2. & Triushnadi Ghrita & Ch. Chi. 26/87-89 \\
\hline 3. & Drakshadya Ghrita & Ch. Chi. 26/93 \\
\hline 4. & Kasherukadi Ghrita & Ch. Chi. 26/94 \\
\hline \multicolumn{3}{|c|}{ Shiro Roga Chikitsa } \\
\hline 1. & Mayur Ghrita & Ch. Chi. 26/163-165 \\
\hline 2. & Mahamayur Ghrita & Ch. Chi. 26/166-174 \\
\hline 3. & Yashtyahwadi Ghrita & Ch. Chi. 26/179 \\
\hline \multicolumn{3}{|c|}{ Vata Vyadhi Chikitsa } \\
\hline 1. & Dashmooladi Ghrita & Ch. Chi. 28/121 \\
\hline 2. & Chitrakadi Ghrita & Ch. Chi. 28/122 \\
\hline 3. & Baladi Ghrita & Ch. Chi. 28/123 \\
\hline \multicolumn{3}{|c|}{ Vata Rakta Chikitsa } \\
\hline 1. & Shravanyadi Ghrita & Ch. Chi. 29/55 \\
\hline 2. & Bala Ghrita & Ch. Chi. 29/56-57 \\
\hline 3. & Parushaka Ghrita & Ch. Chi. 29/58-60 \\
\hline 4. & Jeevaniya Ghrita & Ch. Chi. 29/61-70 \\
\hline 5. & Sthiradi Ghrita & Ch. Chi. 29/76-78 \\
\hline 6. & Padmakadi Ghrita & Ch. Chi. 29/146 \\
\hline \multicolumn{3}{|c|}{ Yonivyapata Chikitsa } \\
\hline 1. & Kashmaryadi Ghrita & Ch. Chi. 30/52-53 \\
\hline 2. & Vrihat Shatavari Ghrita & Ch. Chi. 30/64-68 \\
\hline 3. & Jeevaniya Ghrita & Ch. Chi. 30/148 \\
\hline
\end{tabular}

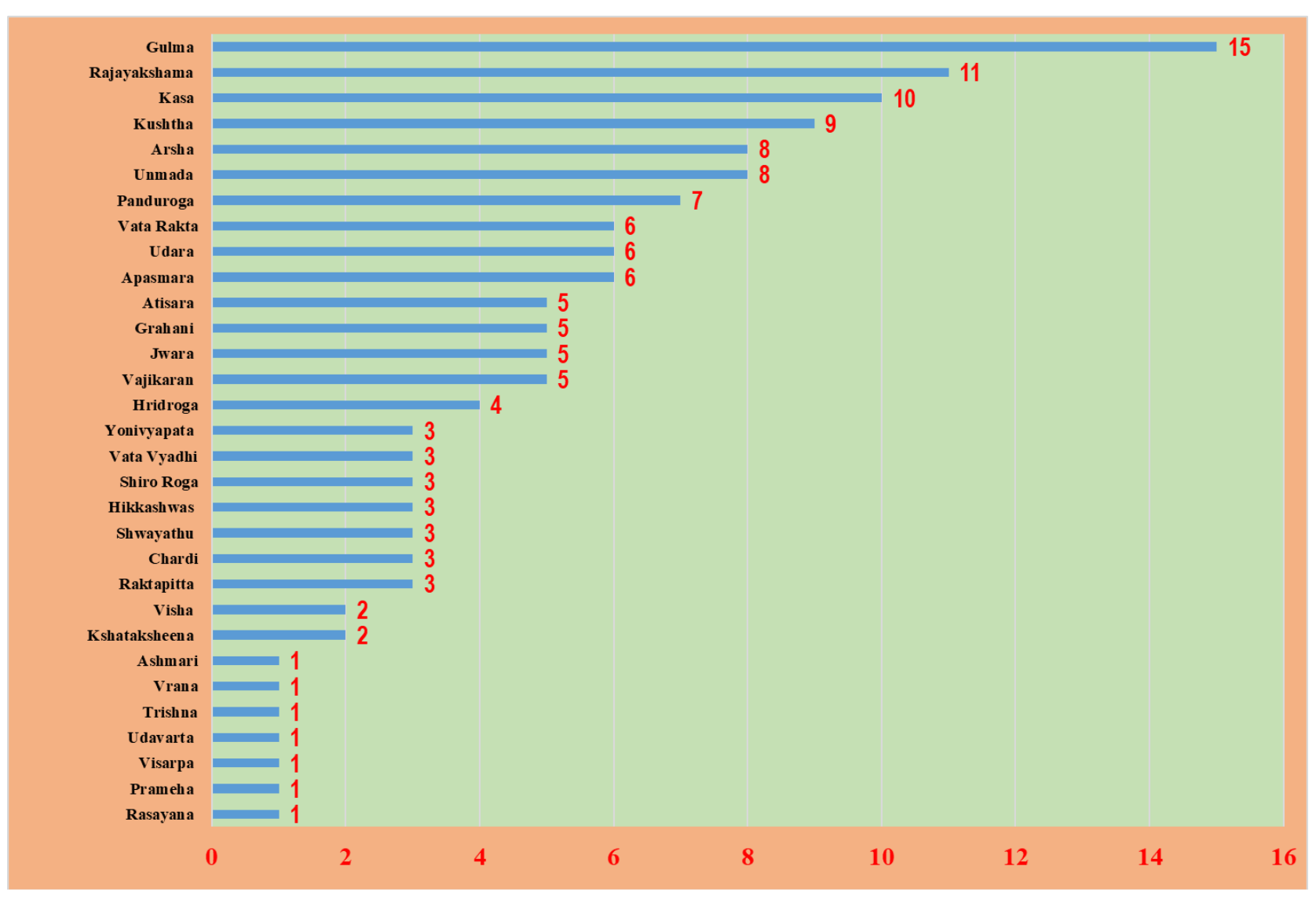

Graph 1: Disease-wise distribution of Ghrita Kalpanas 


\section{DISCUSSION}

In Ayurveda, Ghrita is considered to be the healthiest source of edible fat with added beneficial properties. Ghrita is said to promote longevity ${ }^{5}$ and protect the body from various diseases. It increases Agni (digestive capacity) and improve absorption and assimilation. It nourishes Dhatus (body's tissues), improves memory and pacifies Vata and Pitta. ${ }^{6}$

References where plain administration of Ghee was prescribed have been omitted in this screening limiting to enlisting only compound formulations. The gathered information reveals that large number of medicated ghee formulations is prescribed for majority of the disease conditions. However, though the use of Ghrita is mentioned, its formulations are not found in the management of Madatyaya and Urustambha. More than 200 Ghrita kalpanas have been mentioned in the classics with an objective to promote health and treat diseases. Ghritas have been implicated in increased prevalence of cardiac diseases. But references of medicated ghee are found in the management of Hridaya $\operatorname{roga}^{7}$ (cardiac diseases) in this classic of Charaka Samhita. Researches of current scenario also do not support harmful effects of Ghrita utilization inferring that medicated fats are therapeutically useful. ${ }^{8}$

The general method of preparation and proportion of ingredients in the manufacturing of Ghritas is not explained in detail at a specific place. However, formulation specific methods have been mentioned at respective instances.

In general, paste and liquids of mentioned drugs were used in the manufacturing. The liquids were single at few instances while multiple at other. The nature of liquid was ranging from plain water to fresh juices (Swarasa) $)^{9}$, decoctions (Kwatha ${ }^{10}$ ) of specified herbs. In addition to such herbal extracts, liquids like meat juice (Mamsa rasa $\left.{ }^{11}\right)$, milk (Kseera $\left.{ }^{12}\right)$, buttermilk $\left(\right.$ Takra $\left.^{13}\right)$, curd $\left(D a d h i^{14}\right)$ have also been used. References were also found where Kanji $^{15}$ (Sour gruel), Mutra ${ }^{16}$ (Urine) of various animals were used as the liquid media. Usually different formulations are prepared using Jala (water) as Drava dravya (extracting liquids). Transfer of the medicinal properties of any Dravya (drug) is done by the virtue of Drava dravya. Use of Dravyas like Kseera, Mutra, Takra, Kanji, etc. in preparation of different formulations infers that the seers were familiar with usage of alkaline and acidic Medias for ideal extraction of useful and needful active molecules from the herbs on scientific basis.

Such examples infer that the pharmaceutical technology during the Vedic/ Samhita period was fairly advanced. The seers were well-versed with different functional groups present in various herbs. They were familiar and knew about the nature/ type of therapeutic molecules that can be extracted through different media like aqueous (Swaras, Rasa, Manda, Peya ${ }^{17}$ ), alkaline $\left(K_{s h a r a}{ }^{18}\right)$, oleaginous (Go-Dugdha ${ }^{19}$, Mahishi Ksheera ${ }^{20}$ Ghritamanda, Purana Ghrita ${ }^{21}$, Mahish Ghrita $^{22}$ ), etc. Using salts $^{23}$ and alkaline powders ${ }^{24}$ in the preparations is also an example of such vision. Use of poisonous drugs ${ }^{25}$ (of schedule E1) in preparations infer the sophisticated and advanced scientific methodologies that nullified possible toxicity of such plants.

\section{CONCLUSION}

This synoptic review reveals significance of Ghrita formulations during the period of Charaka Samhita. The information collected in the screening reveals that the concepts of fat-soluble drugs, their utilization in therapeutics were well established during the period of Charaka Samhita. For thousands of years Ayurveda has considered Ghrita as the healthiest source of edible fat. Though many researches of the current times support the beneficial effects of Ghrita emphasized in Ayurvedic classical literature and their utilization as therapeutic agents in the management of various diseases, still there is a need to understand the mode of action of all such formulations in the management of different pathologies.

\section{REFERENCES}

1. Pandit Parashuram Shastri, editor. Adhamalla and Kashirama Commentary on Sharangadhara Samhita, Madhyama Khanda of Sharangadhara, chapter 1, Verse 1, Chaukhamba Publication; New Delhi; 2013. p. 137.

2. G Prabhakar Rao, editor. A Textbook of Bhaisajya Kalpana Vijnanam. Ch. 8, Reprint edition. Chaukhamba Sanskrit Sansthan, New Delhi; 2016. p. 250.

3. Pandit Parashuram Shastri, editor. Adhamalla and Kashirama Commentary on Sharangdhara Samhita, Madhyama Khanda of Sharangadhara, chapter 9, Verse 1, Chaukhamba Publication; New Delhi; 2013. p. 212.

4. Pt. Kashinath Shastri, Dr. Gorakhnatha Chaturvedi, editor. Charaka Samhita of Agnivesha, Chikitsa Sthana. Ch. 1-30, Reprint edition, Chaukhambha Bharati Academy, Varanasi; 2016.

5. Pt. Kashinath Shastri, Dr. Gorakhnatha Chaturvedi, editor. Charaka Samhita of Agnivesha, Sutra Sthana, Ch. 13, Verse 42, Reprint edition, Chaukhambha Bharati Academy, Varanasi; 2016.

6. Pt. Kashinath Shastri, Dr. Gorakhnatha Chaturvedi, editor. Charaka Samhita of Agnivesha, Sutra Sthana, Ch. 27, Verse 232, Reprint edition, Chaukhambha Bharati Academy, Varanasi; 2016.

7. Pt. Kashinath Shastri, Dr. Gorakhnatha Chaturvedi, editor. Charaka Samhita of Agnivesha, Chikitsa Sthana. Ch. 26, Verse: 83, 87-89, 93, 97; Reprint edition, Chaukhambha Bharati Academy, Varanasi; 2016.

8. Joshi KS. Docosahexaenoic acid content is significantly higher in ghrita prepared by traditional Ayurvedic method. J. Ayurved Integr Med 2014; 5: 85-8.

9. Pt. Kashinath Shastri, Dr. Gorakhnatha Chaturvedi, editor. Charaka Samhita of Agnivesha, Chikitsa Sthana. Ch. 2, Verse 1/35, Reprint edition, Chaukhambha Bharati Academy, Varanasi; 2016.

10. Pt. Kashinath Shastri, Dr. Gorakhnatha Chaturvedi, editor. Charaka Samhita of Agnivesha, Chikitsa Sthana. Ch. 11, Verse 50-55, Reprint edition, Chaukhambha Bharati Academy, Varanasi; 2016.

11. Pt. Kashinath Shastri, Dr. Gorakhnatha Chaturvedi, editor. Charaka Samhita of Agnivesha, Chikitsa Sthana. Ch. 8, Verse 93, Reprint edition, Chaukhambha Bharati Academy, Varanasi; 2016.

12. Pt. Kashinath Shastri, Dr. Gorakhnatha Chaturvedi, editor. Charaka Samhita of Agnivesha, Chikitsa Sthana. Ch. 4, Verse 95, Reprint edition, Chaukhambha Bharati Academy, Varanasi; 2016.

13. Pt. Kashinath Shastri, Dr. Gorakhnatha Chaturvedi, editor. Charaka Samhita of Agnivesha, Chikitsa Sthana. Ch. 12, Verse 58-59, Reprint edition, Chaukhambha Bharati Academy, Varanasi; 2016.

14. Pt. Kashinath Shastri, Dr. Gorakhnatha Chaturvedi, editor. Charaka Samhita of Agnivesha, Chikitsa Sthana. Ch. 14, Verse 106, Reprint edition, Chaukhambha Bharati Academy, Varanasi; 2016.

15. Pt. Kashinath Shastri, Dr. Gorakhnatha Chaturvedi, editor. Charaka Samhita of Agnivesha, Chikitsa Sthana. Ch. 9, Verse 52-56, Reprint edition, Chaukhambha Bharati Academy, Varanasi; 2016.

16. Pt. Kashinath Shastri, Dr. Gorakhnatha Chaturvedi, editor. Charaka Samhita of Agnivesha, Chikitsa Sthana. Ch. 9, Verse 
34, Reprint edition, Chaukhambha Bharati Academy, Varanasi; 2016.

17. Pt. Kashinath Shastri, Dr. Gorakhnatha Chaturvedi, editor. Charaka Samhita of Agnivesha, Chikitsa Sthana. Ch. 19 Verse 80-81, Reprint edition, Chaukhambha Bharati Academy, Varanasi; 2016.

18. Pt. Kashinath Shastri, Dr. Gorakhnatha Chaturvedi, editor. Charaka Samhita of Agnivesha, Chikitsa Sthana. Ch. 5, Verse 105, Reprint edition, Chaukhambha Bharati Academy, Varanasi; 2016.

19. Pt. Kashinath Shastri, Dr. Gorakhnatha Chaturvedi, editor. Charaka Samhita of Agnivesha, Chikitsa Sthana. Ch. 5, Verse 74, Reprint edition, Chaukhambha Bharati Academy, Varanasi; 2016.

20. Pt. Kashinath Shastri, Dr. Gorakhnatha Chaturvedi, editor. Charaka Samhita of Agnivesha, Chikitsa Sthana. Ch. 16, Verse 53, Reprint edition, Chaukhambha Bharati Academy, Varanasi; 2016.

21. Pt. Kashinath Shastri, Dr. Gorakhnatha Chaturvedi, editor. Charaka Samhita of Agnivesha, Chikitsa Sthana. Ch. 16, Verse 52, Reprint edition, Chaukhambha Bharati Academy, Varanasi; 2016.
22. Pt. Kashinath Shastri, Dr. Gorakhnatha Chaturvedi, editor. Charaka Samhita of Agnivesha, Chikitsa Sthana. Ch. 23, Verse 241, Reprint edition, Chaukhambha Bharati Academy, Varanasi; 2016.

23. Pt. Kashinath Shastri, Dr. Gorakhnatha Chaturvedi, editor. Charaka Samhita of Agnivesha, Chikitsa Sthana. Ch. 5, Verse 69-70, Reprint edition, Chaukhambha Bharati Academy, Varanasi; 2016.

24. Pt. Kashinath Shastri, Dr. Gorakhnatha Chaturvedi, editor. Charaka Samhita of Agnivesha, Chikitsa Sthana. Ch. 5, Verse 142, Reprint edition, Chaukhambha Bharati Academy, Varanasi; 2016.

25. Pt. Kashinath Shastri, Dr. Gorakhnatha Chaturvedi, editor. Charaka Samhita of Agnivesha, Chikitsa Sthana. Ch. 6, Verse 38-39, Reprint edition, Chaukhambha Bharati Academy, Varanasi; 2016.

\section{Cite this article as:}

Anubha Yadav. Formulations of Ghrita in Charaka Samhita: A Synoptic View. Int. J. Res. Ayurveda Pharm. 2020;11(2):123-128 http://dx.doi.org/10.7897/2277-4343.110244

Source of support: Nil, Conflict of interest: None Declared

Disclaimer: IJRAP is solely owned by Moksha Publishing House - A non-profit publishing house, dedicated to publishing quality research, while every effort has been taken to verify the accuracy of the content published in our Journal. IJRAP cannot accept any responsibility or liability for the site content and articles published. The views expressed in articles by our contributing authors are not necessarily those of IJRAP editor or editorial board members. 